\title{
PREPARATION OF HYBRID POLYMERS AND USE AS CONTROLLED RELEASE DELIVERY VEHICLE FOR VOLATILE REPELLENT TO CONTROL Aedes aegypti
}

\author{
Ygor M. de Oliveira ${ }^{\mathrm{a}}$, Ângladis V. Delfino ${ }^{\mathrm{a}}$, Cenira M. de Carvalho ${ }^{\mathrm{a}}$, Lucia M. Conservaa and Fabiane C. de Abreu ${ }^{\mathrm{a}, *, \odot}$
}

anstituto de Química e Biotecnologia, Universidade Federal de Alagoas, 57072-900 Maceió - AL, Brasil

Recebido em 16/09/2020; aceito em 30/11/2020; publicado na web em 20/01/2021

\begin{abstract}
The Zika virus is a flavivirus spread by the Aedes aegypti mosquito, which also transmits dengue and chikungunya. Current forms of control include elimination of mosquito breeding sites, use of insecticides, repellents and other means. The present study developed new adsorbent materials based on silica and biomaterials capable of releasing an Aedes aegypti mosquito repellent. The materials synthesized were characterized by Fourier-Transform Infrared, thermogravimetric analysis, Scanning Electron Microscopy and Brunauer, Emmett and Teller analysis and tested with citronella, an essential oil widely recognized as a natural insect repellent. The materials presented type IV isotherms, with type H1 hysteresis, characteristic of mesoporous materials, with pore diameters of between $50 \AA$ and $80 \AA$. Materials based on silica-chitosan-glutaraldehyde dried in an oven (SCGO) and silica-chitosanglutaraldehyde dried under a vacuum (SCGV) were found to produce more controlled release of citronella essential oil. The bioassay test was performed using a horizontal double-choice olfactometer. The adsorbent SCGO remained stable for the four hours of the bioassay, while the commercial repellent became ineffective after two hours of release. The production of a prototype in the form of bracelets, stickers releasing a volatile repellent is therefore proposed as a method for the control of the mosquito in urban areas.
\end{abstract}

Keywords: adsorbents; volatile repellents; biopolymers.

\section{INTRODUCTION}

The Aedes (Stegomyia) aegypti Linnaeus mosquito is a cosmopolitan species that is present mainly in tropical and subtropical regions, where climatic and social conditions favor its development. It is found in all the States that make up the Brazilian Federative Republic and in approximately 4,500 municipalities in Brazil. For reason of its extensive global distribution, it is involved in the transmission of various arbovirus diseases, such as Zika, dengue, yellow fever and chikungunya.

The Zika virus is a flavivirus (Flaviviridae family) that was first isolated from a febrile female Rhesus monkey in the Zika Forest, located near Entebbe in Uganda, from which the virus takes its name. ${ }^{2}$ This virus is related to the yellow fever and dengue virus, which are also transmitted by the Aedes aegypti mosquito and cause hemorrhagic fever. The Zika virus causes fever, accompanied by other mild general symptoms, such as headache, rash, malaise, edema and sometimes severe joint pain. However, despite the apparent benign nature of this disease, more severe conditions, including central nervous system involvement (Guillain-Barré syndrome, transverse myelitis and meningitis) have recently been reported to be associated with Zika in French Polynesia and Brazil. This shows how little is known about this disease. The World Health Organization declared the Zika virus outbreak in the Americas a global emergency. This followed the rapid emergence of the virus in Brazil and the South American subcontinent and recognition of a link with the rise in the number of babies born with microcephaly and other neurological abnormalities. ${ }^{3}$

Dengue fever has been a public health problem in Brazil for many years. Since 2015, Zika and chikungunya have also emerged and have caused deaths and serious health problems among the Brazilian population. The main strategy adopted to combat these diseases is extensive use of insecticides and larvicides. These, however, have been shown to be ineffective and are also toxic. There is hope of development of a vaccine against Zika, but it may take years for such

*e-mail: caxico.fabiane@gmail.com a vaccine to be available for use. In consequence of this, there is an ongoing search for new simple fast strategies for elimination of the Zika vector mosquito, since many deaths have been registered, and the quality of life of many impaired. This is especially the case among those afflicted with chikungunya, which can remain debilitating for months or even years after initial infection.

In view of the damage to human health caused by Ae. aegypti, numerous studies have been conducted to find alternative ways to control this vector. These include the use of plant repellents, to prevent mosquito bites and consequently the transmission of the arboviruses that cause disease. ${ }^{4-6}$

The degree of efficacy of repellents depends greatly on the composition of the product. Formulations based on creams, polymer blends or microcapsules for controlled release increase the duration of repellence activity. ${ }^{7}$ Polymers, such as activated carbon, Porapak Q, Tenax GR or Tenax TA, are used in analyses of the retention of volatile organic compounds. ${ }^{8,9}$ Chitosan is another polymer that is widely used in research, as it possesses excellent biodegradation properties and is a naturally occurring polysaccharide. ${ }^{10}$ This substance has produced excellent results against insects, with studies showing that the most active derivative of chitosan (N- (2-chloro-6-fluorobenzyl), when incorporated into an artificial diet, caused $100 \%$ mortality of larvae. ${ }^{11}$ Nanoparticles of chitosan synthesized from shrimp and crab exoskeletons incorporated in a solution tested in a larvicidal bioassay against Ae. aegypti have produced mortality of $98 \%$ and $93 \%$ respectively at a concentration of $100 \mathrm{mg}$. $\mathrm{L}^{-1}{ }^{12}$

Chitosan has also been used with technology relating to the microencapsulation of the essential oils of plants, to reduce loss through evaporation. For reason of its high volatility, this technique is commonly used in the processing of repellents containing citronella essential oil (Cympobogon spp.) Organic-inorganic hybrids are materials formed by the combination of organic and inorganic components that produce materials with characteristics different from those of their components but that reflect the chemical nature of both, combining the thermal stability of inorganic materials with the chemical characteristics of organic compounds. ${ }^{13}$ The present study 
thus aimed to develop Aedes aegypti control strategies that are less harmful to the environment and present greater stability.

\section{EXPERIMENTAL}

The present study synthesized the adsorbents, investigated their adsorptive properties, and measured citronella essential oil release. All reagents used were of analytical grade and deionized water $(18 \mathrm{M} \Omega \mathrm{cm})$ was obtained using a Millipore Inc Milli-Q purification system.

\section{Synthesis of silica-chitosan-glutaraldehyde}

For the preparation of the SCG material, 2.0 grams of chitosan were solubilized in $500 \mathrm{ml}$ of $2 \%(\mathrm{v} / \mathrm{v}$ ) acetic acid. Ten grams of silica gel were then added to the chitosan solution, followed by $100 \mathrm{ml}$ of glutaraldehyde $20 \%$. After filtration, the solid material was washed with deionized water to remove excess glutaraldehyde, leaving no trace of this compound. The material was then divided into two fractions and dried using two different methods. The first fraction was distributed in a Petri dish, which was subsequently placed in a desiccator with a suction pump for 24 hours. This was called silicachitosan-glutaraldehyde dried under vacuum (SCGV). The second fraction was heated in an oven at $50{ }^{\circ} \mathrm{C}$ for 24 hours. This fraction was called silica-chitosan-glutaraldehyde dried in an oven (SCGO).

\section{Characterization}

The synthesized materials (SCGV and SCGO), chitosan, silica gel and glutaraldehyde were characterized by Fourier Transform Infrared Spectroscopy (FTIR) according to the methodology adapted by Silva et al. ${ }^{14}$ in order to identify organic functional groups that may act as adsorption sites. The areas of the materials were determined using the method developed by Brunauer, Emmett and Teller (BET), whereby the adsorption and desorption isotherms are calculated at the temperature of liquid nitrogen $\left(-196^{\circ} \mathrm{C}\right)$. The FTIR spectra were obtained by attenuated total reflection (ATR) carried out at the Center for Analysis of the UFAL's Institute of Chemistry and Biotechnology.

Thermogravimetric (TG) analyses were also performed to evaluate the thermal behavior of the adsorbent materials. ${ }^{15}$ These analyses were performed in a temperature range of $25-800{ }^{\circ} \mathrm{C}$ with a heating rate of $10{ }^{\circ} \mathrm{C} \mathrm{min}^{-1}$ and a dynamic nitrogen atmosphere $\left(20 \mathrm{~mL} \mathrm{~min}^{-1}\right)$ at the Catalysis and Fuels Laboratory of the UFAL's
Department of Chemical Engineering. Finally, the physical structure of the material was determined using a Scanning Electron Microscope (SEM) in order to observe the morphology and any chemical or physical modification of the adsorbents.

\section{Tests of release of citronella essential oil}

The citronella essential oil was first placed beneath the materials using an automatic pipette and analytical balance, to obtain a concentration of $5 \%(\mathrm{~m} / \mathrm{m})$. The release studies were performed using thermogravimetric (TG) analyses with a $40{ }^{\circ} \mathrm{C}$ isotherm and a dynamic atmosphere of $20 \mathrm{~mL} / \mathrm{min}$ of nitrogen. The release test was also performed using an analytical balance over a period of eight days.

\section{Y-tube olfactometer bioassays}

The durability of the behavior of mosquitoes in response to repellent treatments was examined in approximately 15-day-old laboratory-bred Aedes aegypti, kept at a uniform temperature of $27 \pm 2{ }^{\circ} \mathrm{C}$. This analysis was carried out using an olfactometer in the form of a Y-glass tube (trunk, $340 \mathrm{~mm}$, arms, $290 \mathrm{~mm}, 45^{\circ}$ angle, internal diameter, $40 \mathrm{~mm}$ ) connected by the arms to silicone hoses connected to a fluxmeter, as shown in Figure 1. The air flow inside the olfactometer, purified by the active carbon filter, was $1200 \mathrm{~mL} \mathrm{~min}^{-1}$. The experiment was carried out in a room with ambient temperature of $27 \pm 2{ }^{\circ} \mathrm{C}$ and relative air humidity of around $80 \%$. It should be noted that the whole system was cleaned with a neutral detergent and distilled water, followed by an ethanol bath, thus ensuring the removal of any impurity or odor that could possibly interfere with the experiment.

The adsorbent SCGO material containing $5 \%(\mathrm{~m} / \mathrm{m})$ of the citronella essential oil was placed in one of the $\mathrm{Y}$ tube connections, and the control, without citronella, as well as the test with the citronella-based commercial repellent at a concentration of $5 \%(\mathrm{~m} / \mathrm{m})$ were placed in the other connection. After installation of the entire olfactometry unit, ten mosquitoes were inserted into the trunk of the olfactometer and these individuals were observed for four hours, in accordance with the protocol developed by Oyarzún et al. ${ }^{16}$ This process was performed in quadruplicate.

Only situations where the mosquito lasted for more than two hours were counted as a positive response. The results were fed into the following equation developed by the WHO: ${ }^{17}$

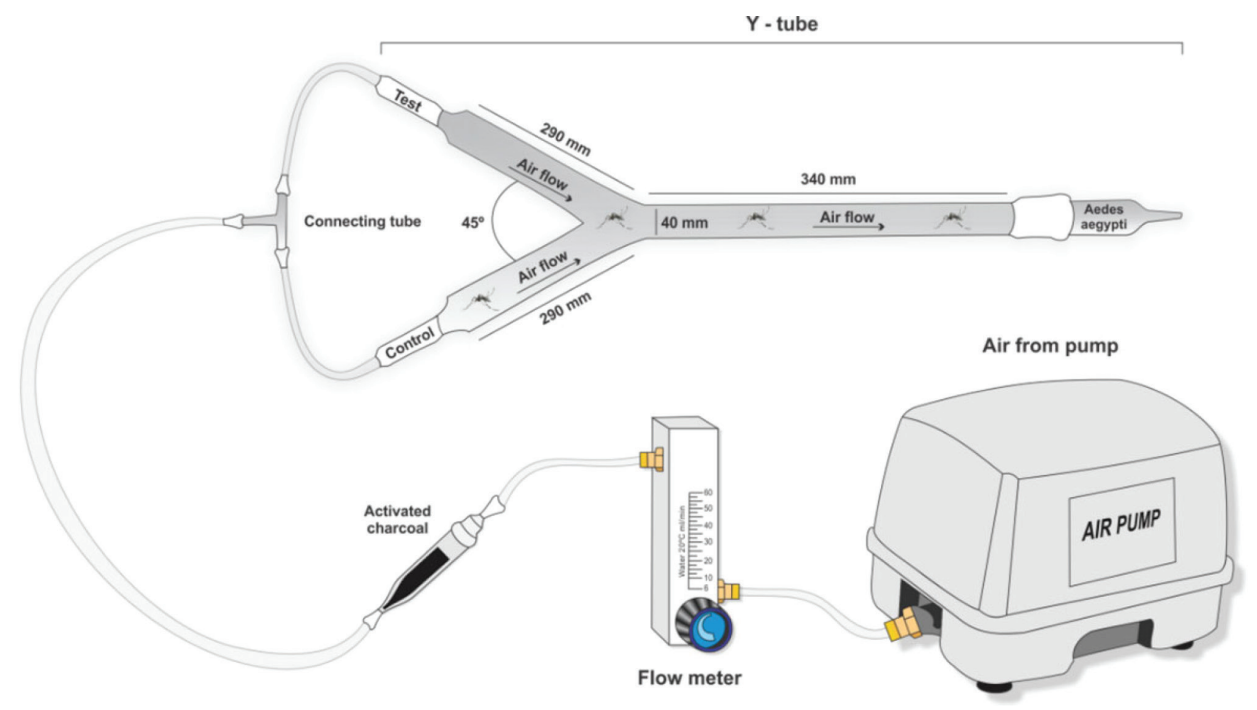

Figure 1. Representation of the Bioassay experiment 

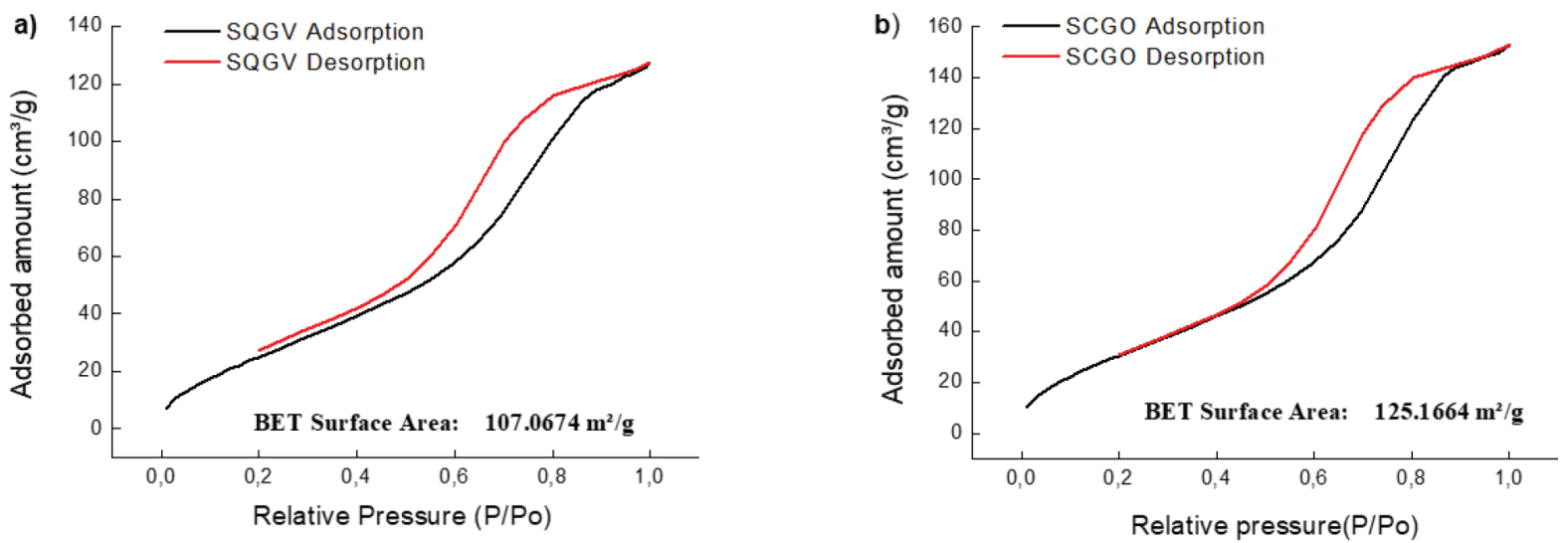

Figure 2. Nitrogen adsorption-desorption isotherms of the synthesized materials (a) SCGV and (b) SCGO

$$
\mathrm{IA}(\%)=100 \times\left[\frac{\mathrm{Ca}-\mathrm{Ta}}{\mathrm{Ca}}\right]
$$

where IA is the percentage of landing inhibition, $\mathrm{Ca}$ is the number of mosquitoes that land in the control space and Ta is the number of mosquitoes that land in the treatment space. The results obtained by the equation were submitted to analysis of variance (ANOVA), followed by the Tukey test with a 5\% level of significance, using the GraphPad Prism 6.0 Program.

\section{RESULTS AND DISCUSSION}

\section{The Brunauer, Emmett and Teller Method}

The surface area, pore volume and distribution of pore sizes for the SCGV and SCGO materials were measured using the Brunauer, Emmett and Teller method (BET) (Figure 2).

The data on the BET-surface areas, pore volumes and pore sizes of the SCGV and SCGO are presented in Table 1. It can be seen that a there was a small variation in surface area according to the drying method used (oven drying or vacuum). There were almost no changes in pore volume. The adsorbents presented pore diameters of between $50 \AA$ and $80 \AA$. According to Sing and colleagues adsorbents with pore diameter of 20 and $500 \AA$ can be classified as mesoporous materials. ${ }^{18}$

The isotherms thus presented characteristics of type IV, with hysteresis of type $\mathrm{H} 1$, with the lower branch representing the quantity of gas adsorbed by increasing relative pressure, while the upper branch represents the amount of gas desorbed by the inverse process. Hysteresis is associated with the differences between the adsorption and desorption processes. Hysteresis type $\mathrm{H} 1$ is characteristic of adsorbents with a narrow and relatively uniform pore distribution. ${ }^{19}$

\section{Spectroscopy in the Fourier-transform infrared region}

Table 2 shows the main vibrational bands of the FTIR spectra of the compounds: (a) Silica, Chitosan, Glutaraldehyde and SCGO; and (b) citronella essential oil (3,7-dimetyl 2,6-octadienal). The silica gel spectrum showed bands at 969 and $1.096 \mathrm{~cm}^{-1}$, related to $\mathrm{Si}-\mathrm{O}$ stretch. ${ }^{20}$ The chitosan spectrum presented bands at $1.560 \mathrm{~cm}^{-1}$, attributed to the deformation of the $\mathrm{NH} 2$ group of the chitosan, and bands at $2.870 \mathrm{~cm}^{-1}$ attributed to the $\mathrm{CH}$ stretch of the chitosan. The bands at 1.030 and $1.150 \mathrm{~cm}^{-1}$ are related to the $\mathrm{C}-\mathrm{O}$ stretch present in the structure. The SCGO spectrum shows the presence of the silica and chitosan bands with small displacements, indicating an interaction between the functional groups present in these compounds and the formation of a new material. The bands at 1.325 and $1.375 \mathrm{~cm}^{-1}$ relate
Table 1. BET surface areas and pore volumes of the SCGV and SCGO materials

\begin{tabular}{lccccccc}
\hline \multirow{2}{*}{ Sample } & \multicolumn{3}{c}{$\mathrm{S}\left(\mathrm{m}^{2} \mathrm{~g}^{-1}\right)$} & & \multicolumn{2}{c}{$\mathrm{V}\left(\mathrm{cm}^{3} \mathrm{~g}^{-1}\right)$} \\
\cline { 2 - 3 } \cline { 7 - 8 } & $\mathrm{S}_{\mathrm{BET}}$ & $\mathrm{S}_{\mathrm{Ex}}$ & $\mathrm{S}_{\text {Micro }}$ & & $\mathrm{V}_{\text {Micro }}$ & $\mathrm{V}_{\text {Total }}$ \\
\hline SCGO & 125 & 183 & 153 & & 0.0329 & 0.2305 \\
SCGV & 107 & 167 & 133 & & 0.0344 & 0.1927 \\
\hline
\end{tabular}

$\mathrm{S}_{\mathrm{BET}}$ : Area by the BET method; $\mathrm{S}_{\mathrm{EX}}$ : External area $\mathrm{S}_{\text {Micro: }}$ Micropore area; $\mathrm{V}_{\text {Micro }}$ : Micropore volume; $\mathrm{V}_{\text {Total }}$ Total volume of pores.

to the stretching of the amide type, which is formed in the reactions of the amine groups of the chitosan with the glutaraldehyde. ${ }^{21}$

Table 2. Main vibrational bands of the silica, chitosan, glutaraldehyde and SCGO materials

\begin{tabular}{lcc}
\hline Material & $\mathrm{v}\left(\mathrm{cm}^{-1}\right)$ & Attribution of the Main Vibrational Bands \\
\hline \multirow{2}{*}{ Silica } & 969 & Si-O Stretching \\
& 1069 & Si-O Stretching \\
\hline \multirow{3}{*}{ Chitosan } & 2870 & C-H Stretching of the Chitosan \\
& 1560 & Deformation of the $\mathrm{NH}_{2}$ group \\
& 1030 & C-O Stretching \\
\hline Glutaraldehyde & 2944 & C-O Stretching \\
\hline \multirow{4}{*}{ SCGO } & 1150 & C-H Propyl Stretching \\
& 1375 & Si-O Stretching \\
& 1030 & Amide Stretching (reaction with \\
& 1150 & glutaraldehyde) \\
& 2870 & C-O Stretching \\
& 2944 & C-O Stretching \\
& & C-H Propyl Stretching \\
\hline
\end{tabular}

The citronella essential oil spectrum, Figure 3, thus shows bands at 2.965 and $2.917 \mathrm{~cm}^{-1}$, indicating the presence of the $\mathrm{CH}_{2}$ group and $\mathrm{CH}_{3}$ aliphatic, as well as the band at $1.455 \mathrm{~cm}-1$ characteristic of the angular deformation $\left(\mathrm{CH}_{2}\right) \mathrm{n}$, when $\mathrm{n}<3$. It also presented the band at $1.377 \mathrm{~cm}^{-1}$ characteristic of the germinal dimethyl angular deformation $\left(\mathrm{CH}_{3}\right)$ and the band at $1.641 \mathrm{~cm}^{-1}$ characteristic of alkene stretching $(-\mathrm{C}=\mathrm{C}-)$. As an aldehyde, citronella essential oil also shows the bands characteristic of this functional group, such as $\mathrm{CH}$ stretching of the aldehyde at $2.715 \mathrm{~cm}^{-1}$ and the band characteristic of the carbonyl group $(\mathrm{C}=\mathrm{O})$ of aldehyde at $1.720 \mathrm{~cm}^{-1} .^{22}$

We also found, using the FTIR technique, that there was an 
interaction between the citronella essential oil and the synthesized materials, as shown in Figure 4. Bands at 2965, 2917 and $2715 \mathrm{~cm}^{-1}$, attributed to the citronella, can be seen on the materials even after eight days of release.

\section{Scanning electron microscopy}

Scanning electron microscopy (SEM) was used for morphological characterization of chitosan, SCGO and SCGV (Figure 5). These images show a clear difference between the chitosan and the prepared materials, with the chitosan exhibiting a rough surface and more porous appearance compared to the other materials. Generally, speaking particles of non-uniform size are found in hybrid materials, as is the case with silica gel. The incorporation of chitosan reduces the amount of empty space between particles. On the other hand, the surface of the SCGO and SCGV materials were found to be similar, as is to be expected when the materials are the same, differing only with respect to the drying method used.

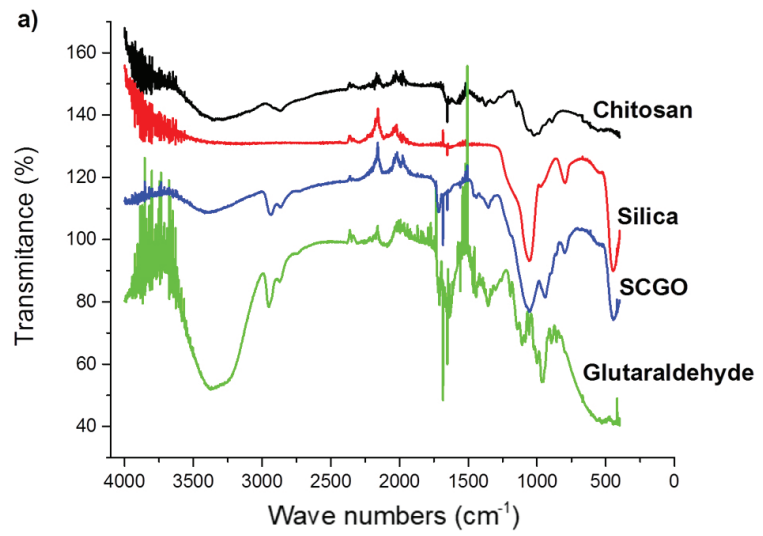

\section{Thermogravimetric analysis}

The thermic behavior of the chitosan, SCGO and SCGV materials was evaluated by thermogravimetric analysis. It has been reported in the literature that the hybrid compounds formed by chitosan and silica present a three-stage pattern of thermal decomposition. The thermogravimetric (TG) and first derivative (DTG) curves for the synthesized materials were obtained and the data on loss of mass are presented in Table 3. TGA results for the chitosan and chitosan with citronella are shown in Supplementary Material. For chitosan alone, there is an initial mass loss of $5 \%$ at $100{ }^{\circ} \mathrm{C}$ related to water loss. In the presence of the oil produced $36 \%$ weight loss in the same temperature range. It is interesting to note that comparing both materials can be observed a weight loss $31 \%$ related to citronella oil adsorbed or absorbed in the chitosan $\left(310 \mathrm{mg} \mathrm{g}^{-1}\right)$.

Evaluation of the TGA and DTG curves of the synthesized materials revealed no differences, suggesting that the materials have similarities with respect to their chemical composition. The

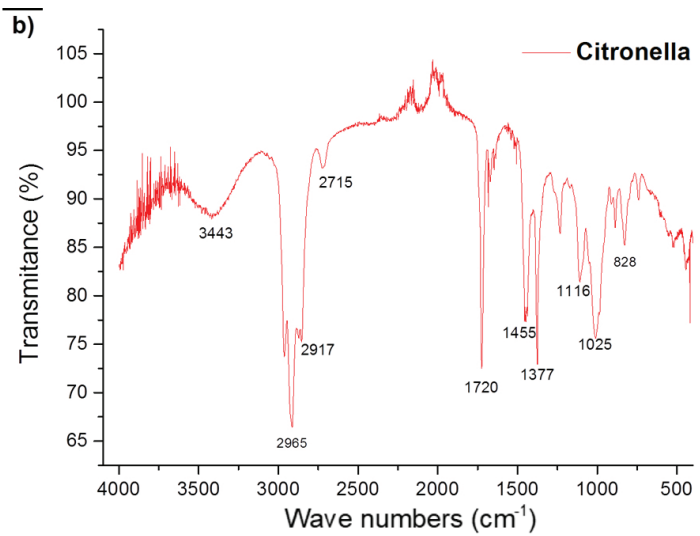

Figure 3. Spectroscopy in the infrared region of the compounds: (a) Glutaraldehyde, Silica, Chitosan and the SCGO material, (b) Citronella essential oil
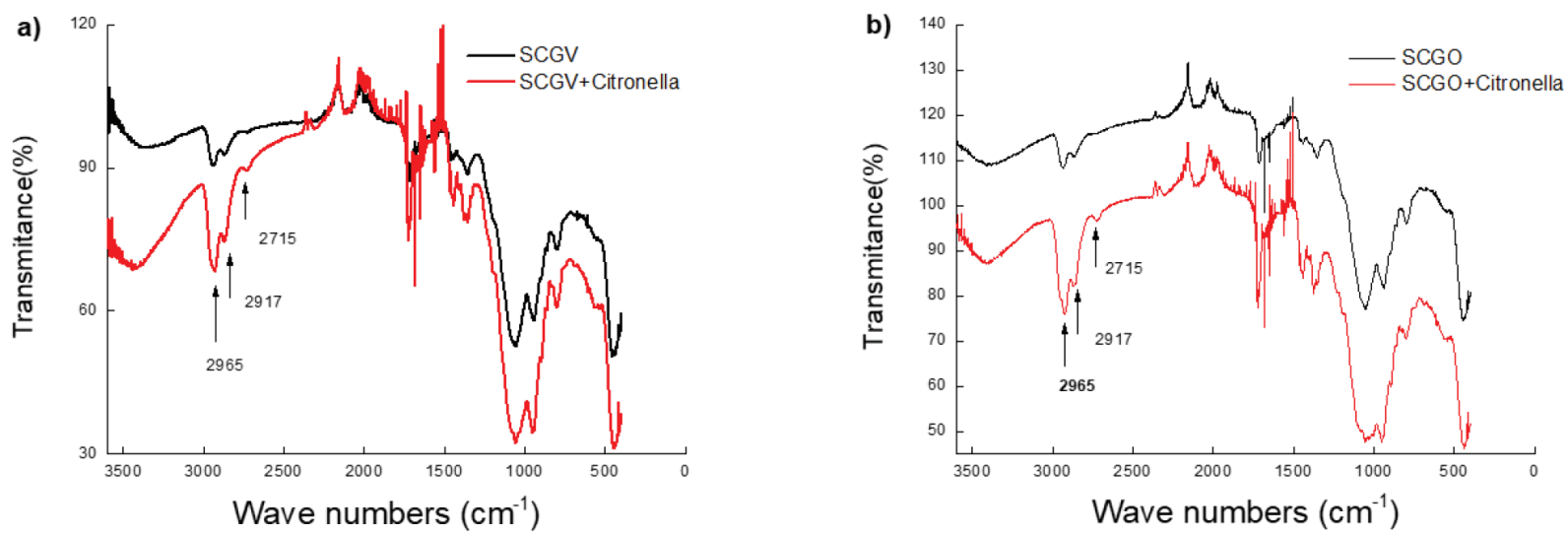

Figure 4. Spectroscopy in the infrared region for the compounds: (a) SCGV and (b) SCGO, in the presence of citronella at 5\%, after 8 days of release
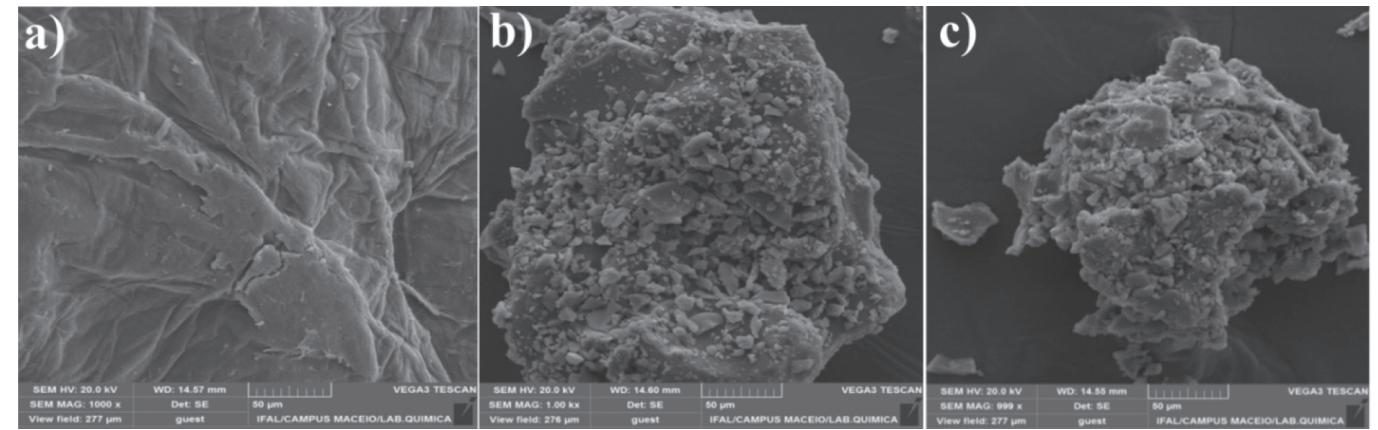

Figure 5. SEM of (a)Chitosan 1000x; (b) SCGO 1000x; (c) SCGV 999x 
Table 3. Data on the mass loss of materials

\begin{tabular}{|c|c|c|c|c|c|c|c|}
\hline \multirow[b]{2}{*}{ Material } & \multicolumn{2}{|c|}{$1^{\text {st }}$ mass loss } & \multicolumn{2}{|c|}{$2^{\text {nd }}$ mass loss } & \multicolumn{2}{|c|}{$3^{\text {rd }}$ mass loss } & \multirow[b]{2}{*}{ TOTAL } \\
\hline & Mass loss (\%) & $\begin{array}{l}\text { Temperature } \\
\text { range }\left({ }^{\circ} \mathrm{C}\right)\end{array}$ & Mass loss $(\%)$ & $\begin{array}{l}\text { Temperature } \\
\text { range }\left({ }^{\circ} \mathrm{C}\right)\end{array}$ & Mass loss (\%) & $\begin{array}{l}\text { Temperature } \\
\text { range }\left({ }^{\circ} \mathrm{C}\right)\end{array}$ & \\
\hline SCGV & 40 & $40-200$ & 15 & $200-260$ & 10 & $300-500$ & $65 \%$ \\
\hline SCGO & 35 & $40-200$ & 15 & $200-260$ & 10 & $300-500$ & $60 \%$ \\
\hline
\end{tabular}

first stage of mass loss occurred between 40 and $200{ }^{\circ} \mathrm{C}$. This event was responsible for the mass loss of $30 \%$ to $45 \%$ of the material, as confirmed by the DTG curve, in which the temperature at which this phenomenon occurs is $135^{\circ} \mathrm{C}$. This can be attributed to the release of residues of acetic acid, glutaraldehyde and citronella physically adsorbed on the surface of the materials.

It is also possible that the silica itself contributes to the initial mass loss peaks of these materials, as a result of residual silane groups present on the surface of the silica gel that have not undergone a reaction, enabling these groups to cause each other to condense, releasing water as volatile product. ${ }^{23}$

The SCGV material presented a larger quantity of volatiles, citronella and adsorbed water, and a greater initial loss of mass was consequently observed. This is caused by the oxygenated and nitrogenous groups present in chitosan, which interact with water through hydrogen bonds. The second mass-loss event corresponds to the decomposition of the chitosan present in large quantities in this material due to the glutaraldehyde cross-linking. In the third loss of mass, the event is attributed to some change in the silica due to the high temperature, presenting a loss of $10 \%$ up to a temperature of $500{ }^{\circ} \mathrm{C}$. It is also possible that a decomposition phase occurs, through the dehydration of saccharide rings, depolymerization and decomposition of the organic polymer units. ${ }^{24}$

Evaluation of the DTA graphs for the SCGO and SCGV materials reveals two distinct events related to mass loss: one with an endothermic profile with a maximum of $135{ }^{\circ} \mathrm{C}$, related to the evaporation of the volatile compounds and another with an exothermic profile, with a maximum of $260{ }^{\circ} \mathrm{C}$ for the degradation of the crosslinked chitosan (Supplementary Material).

\section{Study of the citronella essential oil release}

The volatilization characteristics of the essential oil adsorbed by different materials were studied using an isothermal system. The mass loss and mass loss rate of citronella for Chitosan and the hybrid material were determined using thermogravimetric analysis similar to that used for analysis of coal and these figures were used to determine release constants.

Initially, the sample was prepared by adding $8 \mathrm{mg}$ of citronella to $20 \mathrm{mg}$ of the adsorbent material. Citronella thus accounted for $28.6 \%$ of the mass of the sample. The studies of citronella essential oil release were performed using isothermal gravimetric analysis at a temperature of approximately $40{ }^{\circ} \mathrm{C}$ (Figure 6a). For chitosan, citronella desorption was greater than for the hybrid material. For the hybrid materials (SCGO and SCGV), the process was found to be linear from 2,000 seconds onwards. The DTA graph showed an event with endothermic characteristics up to 2,000 seconds, indicating that the volatile repellent is absorbed by the material. However, as our interest is in adsorbed content, a curve fitting was obtained for 2,000 seconds onwards to ensure that no event influenced the linearity of the release curves.

Considering the curve only after 2,000 seconds, where the process of citronella desorption occurs in a more linear fashion, characteristic of zero-order reactions, the constant was calculated for all materials synthesized using the graph method (Figures $6 \mathrm{~b}$ and $6 \mathrm{c}$ ), where $\mathrm{k}$ corresponds to the slope of the curve.

$$
[\mathrm{A}]=-\mathrm{kt}+[\mathrm{A}]_{0}
$$

The release rate at $40{ }^{\circ} \mathrm{C}$ for each material was calculated based on the mass of citronella adsorbed and whether release occurred between 2000 and 7000 seconds (0 to 3000 seconds for chitosan). For chitosan, citronella desorption was greater than for the hybrid material. For hybrid materials the release rate was similar (Table 4).

Analysis of the mass loss release curve in an analytical balance for 8 days revealed similar behavior. The curve became more linear from the $2^{\text {nd }}$ day onwards, a find which is related to the quantity of mass adsorbed by the material. Mass released before the $2^{\text {nd }}$ day represents non-absorbed compound. This is shown by the abrupt decrease in the curve at the beginning of the experiment. As it exhibited a similar release behavior and was obtained using a simpler drying process, only adsorbent material SCGO was used in the bioassay studies

\section{Bioassay}

At the beginning of the experiment, it was common for the mosquitoes to appear agitated. This was probably because of the release of the citronella (C) absorbed in the material, which tends to be released quickly at the beginning of the process, as observed in the TG analysis.

The results obtained in the present study demonstrate the efficiency of the SCGO material in the presence of citronella in inhibiting Ae. aegypti for several hours. Figure 7 shows that there were no significant differences between the treatments used for the period of one hour, indicating that all treatments are initially efficient repellents. However, after two hours of bioassay, there was a significant difference between $\mathrm{SCGO}+\mathrm{C}$ and the commercial repellent $\mathrm{RC}(\mathrm{P}<0.01)$, as well as between $\mathrm{SCGO}+\mathrm{C}$ and SCGO $(\mathrm{P}<0.05)$, but no difference between SCGO and RC. Behavior after two hours was found to be similar to that after two hours. It can thus be concluded that the commercial repellent and the product without citronella do not provide any increase in inhibition compared to the product containing citronella. After four hours of treatment, a significant difference was observed between the $\mathrm{SCGO}+\mathrm{C}$ sample and the $\mathrm{RC}(\mathrm{P}<0.01)$, as well as between SCGO and RC, but there was no significant difference between SCGO+C and SCGO. This suggests that the commercial repellent is not efficient.

The data obtained showed that, over the full four hours of the experiment, the $\mathrm{SCGO}+\mathrm{C}$ treatment had a better rate of inhibition of the mosquito, with $100 \%$ inhibition in the first hour and $97.25 \%$ in the last. The SCGO treatment had the highest inhibition rate in the first hour of the experiment (72.91\%), showing it to be the second best alternative for inhibition of Ae. aegypti, In the last hour there was a slight reduction in inhibition to $72.13 \%$ for this treatment. Although SCGO without citronella presented the second highest rates (in percentage) of inhibition to the mosquito, this treatment only differed from the commercial repellent in the last hour of the experiment, 

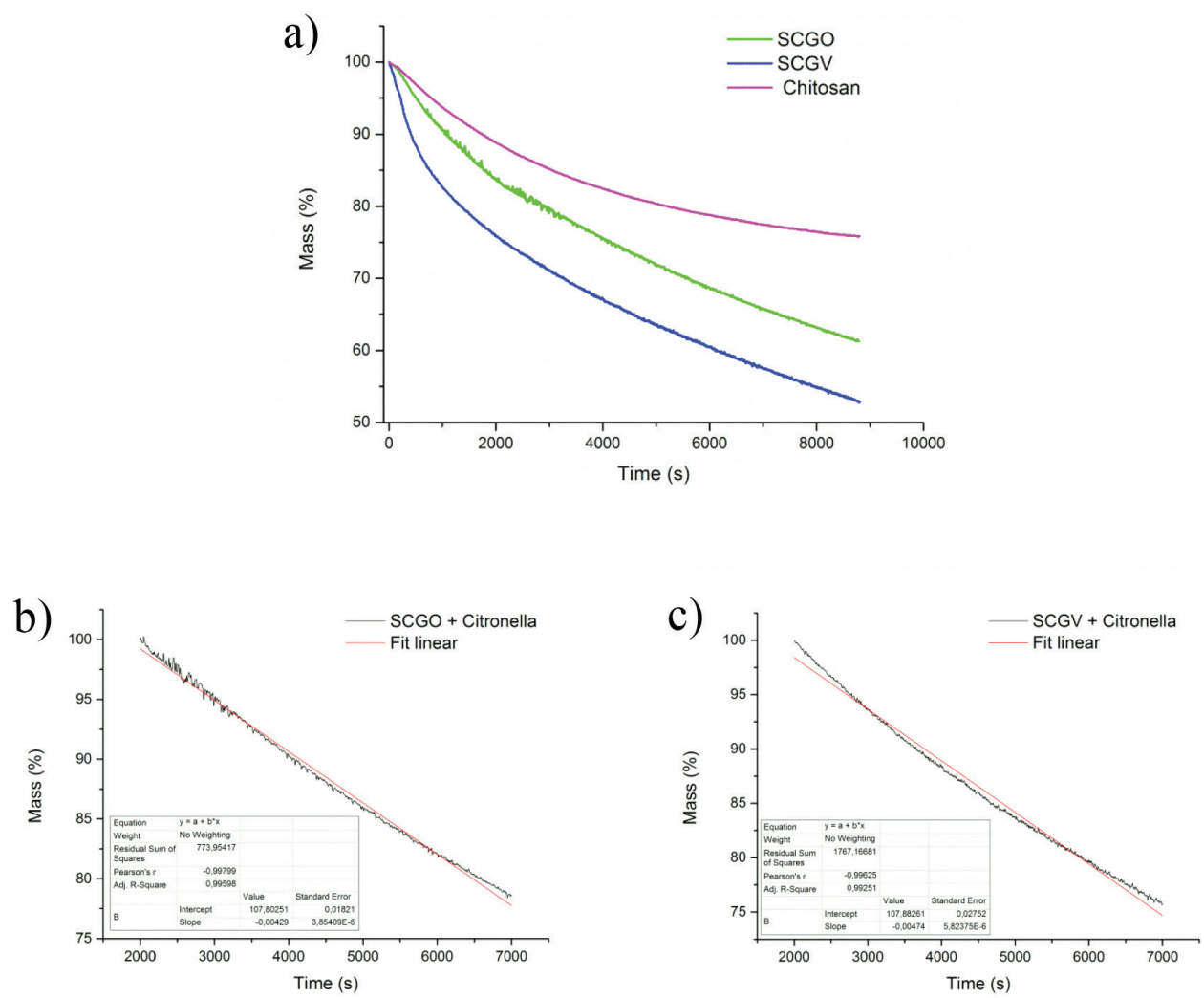

Figure 6. a) Thermogravimetric release curves for the chitosan, SCGO, SCGV samples, with citronella; curves for the volatile repellent (2000-7000 seconds) with their respective linear regressions b) SCGO and C) SCGV

Table 4. Release constants values hybrid materials

\begin{tabular}{ccc}
\hline Material & $\begin{array}{r}\text { Release rate }\left(\mathrm{mg} \mathrm{h}^{-1}\right) \\
\text { at } 40{ }^{\circ} \mathrm{C}\end{array}$ & $\begin{array}{c}\text { Constant } \\
\left(\% \mathrm{~s}^{-1}\right)\end{array}$ \\
\hline Chitosan & 0.94 & - \\
SCGO & 0.49 & $3.85 \times 10^{-6}$ \\
SCGV & 0.49 & $5.82 \times 10^{-6}$ \\
\hline
\end{tabular}

in which it demonstrated greater efficacy. The commercial insect repellent was generally less effective than the other treatments, with inhibition rates of $63.09 \%, 66.33 \%, 46.66 \%$ and $36.66 \%$ for $1,2,3$ and 4 hours duration, respectively, indicating a decline in efficiency over time. The results obtained for the SCGO material developed in the present study concur with the findings of Harismah et al. in a study involving a commercially available repellent paint with the addition of citronella oil and clover leaf, in which they found that, after a period of five hours, there was a tendency for efficiency to decrease. ${ }^{25}$

According to Rodriguez et al., the protection provided by a commercial repellent (RC) based on citronella oil (10\%) has an estimated duration of two hours. ${ }^{26}$ The same authors found that the repellents tested provided maximum protection for two hours. This was then followed by a gradual decline in efficacy up to the conclusion of the four-hour experiment. The findings of this study, however, show that the $\mathrm{SCGO}+\mathrm{C}$ material is a more effective repellent even with a lower proportion of citronella (5\%). Mendki et al. in bioassays to test the activity of formulated repellents containing $20 \%$ of a commercial synthetic repellent DEPA (N, $\mathrm{N}$-diethyl phenyl acetamide) + one plant essential oil against Aedes aegypti, one of these being citrine, found that the average protection time for this formulation was 2.5 hours. ${ }^{27}$

In a study of the effect of essential oils on the behavior of the Aedes aegypti mosquito involving an olfactometer bioassay, Uniyal et $a l$. found that the essential oils of some plants such as litsea, rosewood and geranium were effective repellents of Ae. aegypti, but that these oils were not as effective as the synthetic insect repellents DEET (N,N Diethyl-m-toluamide) and DEPA. ${ }^{28}$ These results contradict the results presented here, in which the commercial repellent was found to be less effective than the $\mathrm{SCGO}+\mathrm{C}$ treatment.

\section{CONCLUSIONS}

The results show that the proposal for synthesis, characterization and study of the release of citronella essential oil by means of adsorptive processes using materials based on chitosan and silica gel was an efficient method. Through the characterization of techniques, it was possible to provide evidence of the formation of the desired materials, especially those relating to the incorporation of chitosan in silica through the glutaraldehyde crosslinking agent. The FTIR spectra and the thermogravimetric curves indicate the incorporation of clusters in the chitosan by way of glutaraldehyde crosslinking, and this produced the best adsorption capacity and release rate, in addition to a satisfactory release constant. In all materials, citronella was present even after eight days of release. SEM results indicate possible sites for adsorption.

The bioassays presented satisfactory results for controlled release of citronella essential oil by means of the adsorbent material SCGO, in which it was stable even after four hours of release.

It will thus be possible to develop a prototype in the form of bracelets or stickers based on the materials developed here, with a view to releasing these volatile compounds in a controlled manner and thereby helping to control the Aedes aegypti mosquito.

\section{SUPPLEMENTARY MATERIAL}

TGA/DTG and DTA curves for Chitosan, Chitosan + Citronella, 

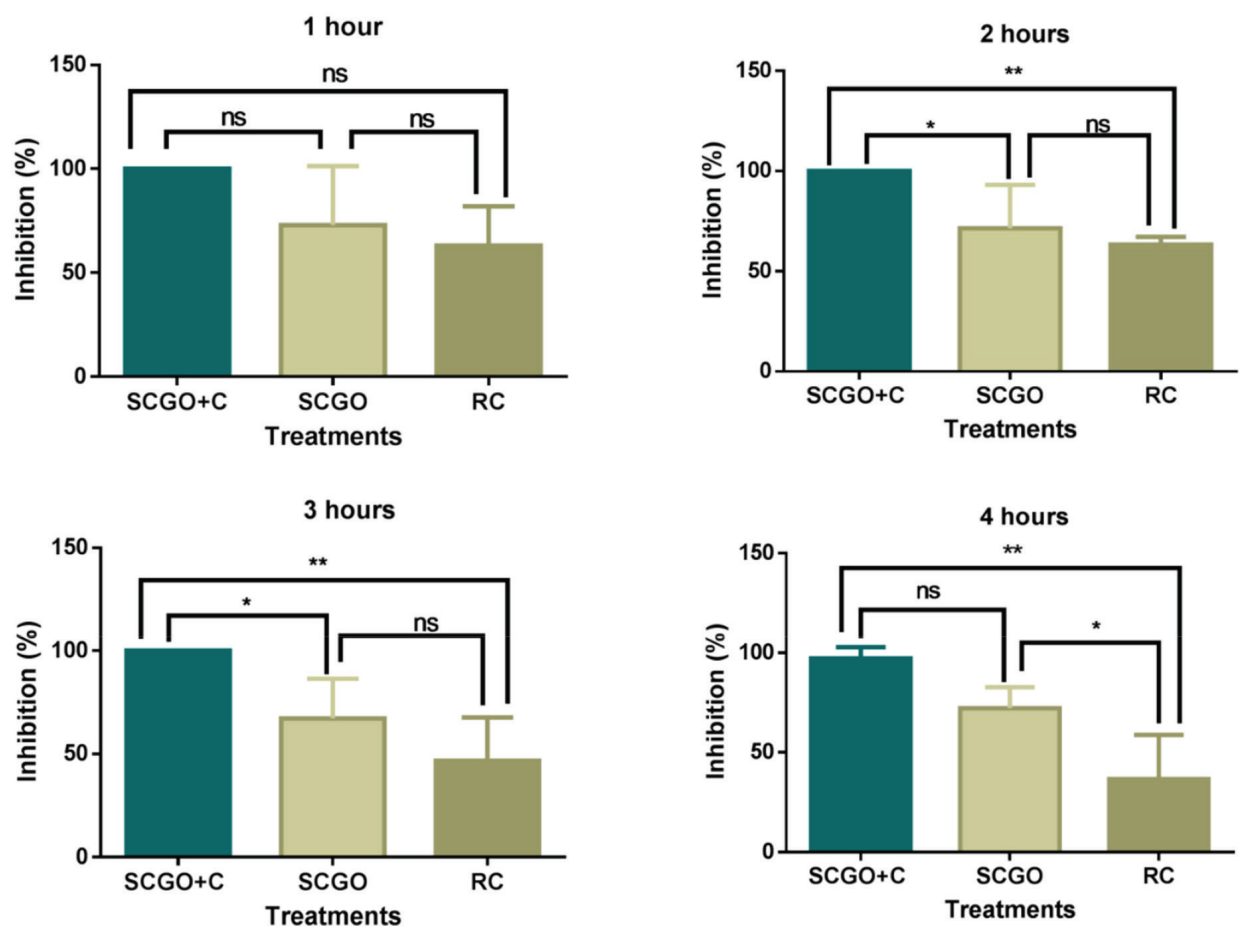

Figure 7. Bioassay using " $Y$ " tube olfactometer to evaluate the duration of inhibition (\%) of samples containing silica, chitosan and glutaraldehyde + citronella $(S C G O+C)$ and SCGO against the Aedes aegypti mosquito compared to synthetic repellent $(R C)$. $P$ values: $P<0.05^{*} ; P<0.01^{* *}$; not significant (ns)

SCGV and SCGO are freely available at http://quimicanova.sbq.org. br, in PDF format.

\section{ACKNOWLEDGEMENTS}

The authors would like to acknowledge funding from the CNPq, Capes and FAPEAL.

\section{REFERENCES}

1. Carvalho, F.D.; Moreira, L.A.; Neotropical Entomology 2017, 46, 13.

2. Karabatsos, N.; American Society of Tropical Medicine and Hygiene $\mathbf{1 9 8 5}, 3,1147$.

3. Petersen, E.; Wilsonb, M. E.; McCloskey, B.; Int. J. Infect. Dis. 2016, $44,11$.

4. Ranasinghe M. S. N.; Arambewela L.; Samarasinghe S.; International Journal of Collaborative Research on Internal Medicine \& Public Health 2016, 8, 40.

5. Diaz, J. H.; Wilderness \& Environmental Medicine 2016, 27, 10.

6. Azeem, M.; Zaman, T.; Tahirb, M.; Harisb, A.; Iqbala, Z.; Binyameenb, M.; Nazire, A.; Ali Shadb, S.; Majeedf, S.; Mozūraitisg, R.; Ind. Crops Prod. 2019, 140 .

7. Nerio, L.S.; Verbel, J.O.; Staashenko, E. Bioresour. Technol. 2010, 101, 1.

8. Brezolin, A. N.; Martinazzo, J.; Muenchen, D. K.; Cezaro, A. M.; Rigo, A. A.; Anal. Bioanal. Chem. 2018, 410, 4091.

9. Bicchi, C.; Maffei, M. Methods Mol. Biol. 2012, 918.

10. Hsieh; W-C; Chang, C-P; Gao, Y-L. Colloids Surf., B 2006, 53.

11. Hadrami, A.E.; Adam, L.R.; Hadrami, I.E.; Daayf, F. Mar. Drugs 2010, 8.

12. Anand, M.; Maruthupandy, M.; Kalaivani, R.; Suresh, S.; Kumaraguru, A. K.; J. Colloid Sci. Biotechnol. 2014, 3, 188.

13. Mladenovska, K.; Cruaud, O.; Richomme, P.; Belamie, E.; Raicki, R. S.; Int. J. Pharm. 2007, 345, 59.
14. Silva, G. S.; Oliveira, P. C.; Giordani, D. S.; Castro, H. F.; J. Braz. Chem. Soc. 2011, 22, 1407.

15. Vasconcelos, H. L.; Camargo, T. P.; Goncalves, N. S.; Neves, A.; Laranjeira, M. C. M.; Fávere, V. T.; React. Funct. Polym. 2008, 68, 572.

16. Oyarzun, M. P.; Palma, R.; Alberti, E.; Hormazabal, E.; Pardo, F.; Birkett, M. A.; Quiroz, A.; J. Med. Entomol. 2009, 46, 1320.

17. Guidelines for efficacy testing of household insecticide products, World Health Organization: Geneva, 2009.

18. Sing, K. S. W.; Adv. Colloid Interface Sci. 1998, 76, 3.

19. Sing, K.S.W.; Everett, D. H.; Haul, R. A. W.; Moscou, L.; Pierotti, R. A.; Rouquerol, J.; Siemieniewska, T.; Pure Appl. Chem. 1985, 57, 603.

20. Mansur, H. S.; Sadahira, C. M.; Souza, A. N.; Mansur, A. A. P.; Mater. Sci. Eng. 2008, 28, 539.

21. Silverstein, R. M.; Webster, F. X.; Kiemle, D. J.; Spectrometric identification of organic compounds, Livros Técnicos e Científicos: Rio de Janeiro, 2007.

22. Cestari, A. R.; J. Colloid Interface Sci. 2005, 292, 363.

23. Jesionowski, T.; Dyes Pigm. 2005, 67, 81.

24. Silva, A. C. O.; Tese de Doutorado, Universidade Federal de Alagoas, Brasil, 2017.

25. Harismah, K.; Vitasari, D.; Mirzaei, M.; Fuadi, A. M.; Aryanto, Y. H.; AIP Conference Proceedings (2017), doi: 10.1063/1.4985468.

26. Rodriguez, S. D.; Drake, L. L.; Price, D. P.; Hammond, J. I.; Hansen, I. A.; J Insect Sci. 2015, 15, 140.

27. Mendki, M. J.; Singh, A. P.; Tikar, S. N.; Parashar, B. D.; Veer, V.; Shukla, S. V.; Prakash, S.; International Journal of Mosquito Research 2015, 2,17

28. Uniyal, A.; Tikar, S. N.; Mendki, M. J.; Singh, R.; Shukla, S. V.; Agrawal, O. P.; Veer, V.; Sukumaran, D.; Journal of Arthropod-Borne Diseases 2016, 10, 372 